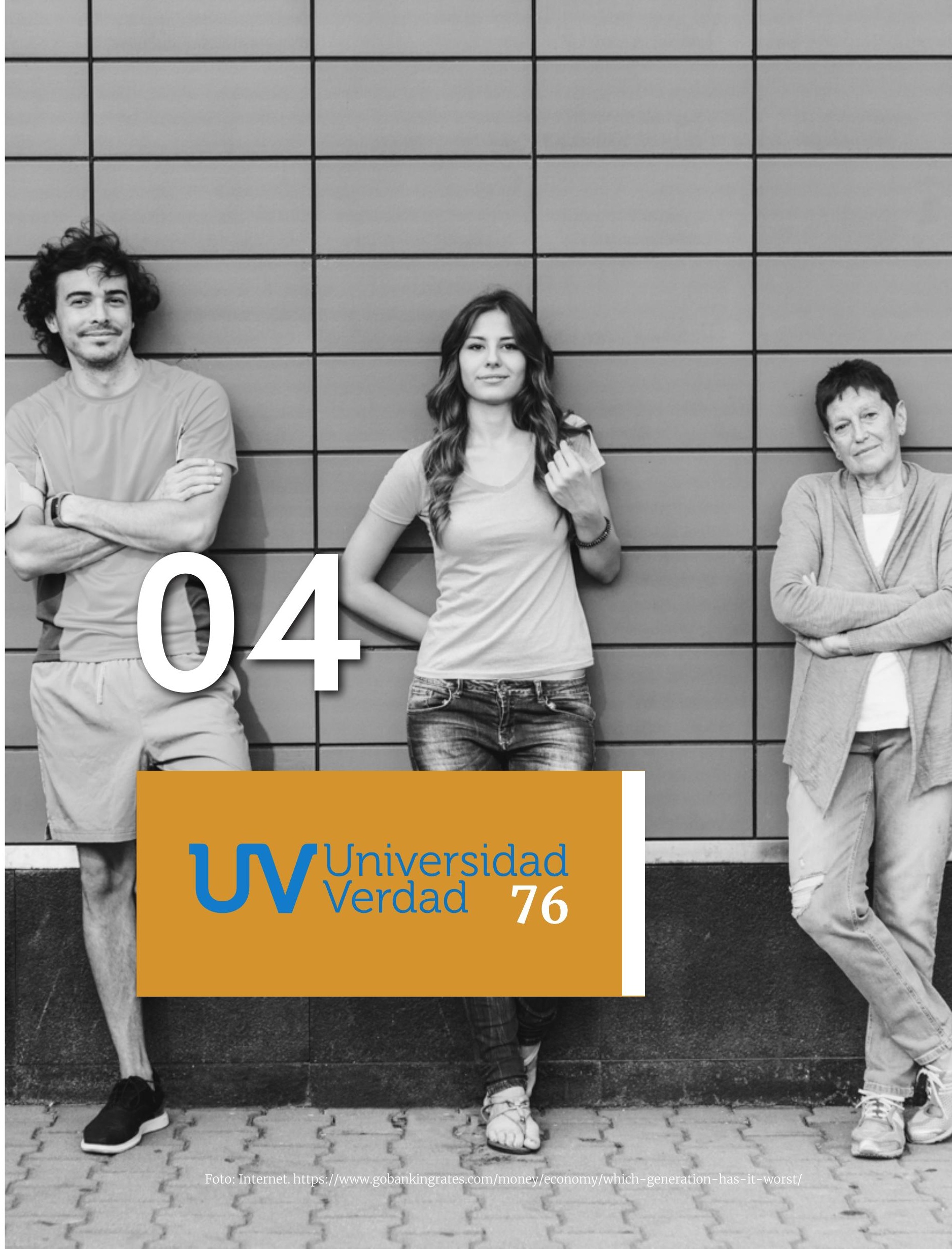




\section{ANÁLISIS DEL GASTO TURÍSTICO EN LOS SEGMENTOS DE MERCADO BABY BOOMERS, GENERACIÓN X Y MILLENNIALS; QUE VISITAN LA CIUDAD DE CUENCA, ECUADOR}

\section{Analysis of tourism spending in the Baby Boomers, Generation $X$ and Millennials market segments; who visit the city of Cuenca, Ecuador}

\footnotetext{
Silvana Astudillo. Profesora de la Facultad de Ciencias de la Hospitalidad, Grupo de Investigación Empresarial y Turístico. Universidad de Cuenca. (Ecuador) (silvana.astudillo@ucuenca.edu.ec) (https://orcid.org/0000-0002-3431-2809)

iD Kevin Maldonado. Grupo de Investigación Empresarial y Turístico. Universidad de Cuenca. (Ecuador) (kevin.maldonado@ucuenca.edu.ec) (https://orcid.org/0000-0001-5691-2015)

iD Chris Maldonado. Grupo de Investigación Empresarial y Turístico. Universidad de Cuenca. (Ecuador) (chris.maldonado0302@ucuenca.edu.ec)

(https://orcid.org/0000-0003-2270-0772)
}

\section{Resumen}

Este artículo tiene como objetivo caracterizar el gasto turístico de manera comparativa en tres segmentos de mercado turístico, basado en la generación a la que pertenecen Baby Boomers, Generación $\mathrm{X}$ y Millennials en la ciudad de Cuenca-Ecuador. Las generaciones están marcadas por periodos de tiempo y grandes cambios en su contexto social: culturales, económicos, tecnológicos, entre otros que provocan diferencias en sus intereses, necesidades y hábitos de consumo. El estudio tiene un alcance descriptivo de corte transversal realizado a 384 turistas. Para la comparación del gasto turístico se utilizó el test estadístico ANOVA y la prueba de comparaciones múltiples de Tukey. Los resultados revelan que existe una diferencia estadística significativa entre los promedios del gasto que realizan los turistas en relación con su generación. En este sentido, entender y caracterizar las distintas generaciones de turistas que visitan la ciudad de Cuenca y contrastarlos entre sí permite generar estrategias y productos específicos para estos mercados, así como el punto de partida para futuras investigaciones.

\section{Abstract}

This paper aims to characterize tourism spending in a comparative way in three segments of the tourism market, based on the generation to which theybelong: Baby Boomers, Generation X and Millennials in the city of Cuenca-Ecuador. The generations are marked by periods of time and major changes in their social context: cultural, economic, technological, among others that causes differences in their interests, needs and consumption habits. This study has a descriptive cross-sectional scope and for the comparison of the tourist expenditure variable applied in 384 tourists. 
The ANOVA statistical test and the Tukey's multiple comparison test were used. The results reveal that there is a significant statistical difference between the average expenditure generated by tourists and their generation. In this sense, understanding and characterizing the different generations of tourists who visit the city of Cuenca and contrasting them with each other allows generating specific strategies and products for these markets, as well as the starting point for future research.

\section{Palabras clave}

Baby Boomers; Generación X; Millennials; Gasto turístico, Cuenca.

\section{Keywords}

Baby Boomers; Generation X; Millennials; tourist expenditure, Cuenca.

1.

\section{Introducción}

Cuenca, la tercera ciudad en importancia del Ecuador, es visitada día a día por distintos tipos de turistas de diferente: edad, procedencia, motivación, gustos y preferencias, lo que genera distintos tipos de demanda y, por ende, distintos niveles de gasto turístico. Esta heterogeneidad, más la falta de información de los visitantes, crea una oferta con servicios generalizados, y no permite aprovechar plenamente el potencial económico derivado del turismo.

Este potencial económico se refleja en la cantidad de dinero que el visitante genera en el lugar de destino, no todos los turistas comparten los mismos hábitos de consumo, por lo que es necesario diferenciar y comprender su gasto dentro de la ciudad, para así generar estrategias que permitan obtener un mayor ingreso y, a la vez, satisfacer las necesidades y expectativas del visitante.

La mercadotecnia cuenta con un conjunto de técnicas enfocadas a los consumidores, una de estas herramientas fundamentales, es la segmentación, que nos permite dividir al mercado en fracciones, con características similares, para entender su comportamiento y la mentalidad a la hora de consumir; todo esto, con el fin de provocar cambios favorables en la conducta de estos segmentos. Así pues, explorar diferentes nichos turísticos y comparar el comportamiento puede ser de mucho interés (Brida y Scuderi, 2013), así como comparar su nivel de gasto (Wang y Davidson, 2010).

Una forma de diferenciar a los segmentos es mediante generaciones: "conjunto(s) de personas $\mathrm{cu}$ yas fechas de nacimiento o edades son próximas y han recibido educación, influencia cultural y/o social semejantes, a su vez, adoptan una actitud y/o pensamiento similar" (Kotler y Keller, 2012). Este tipo de segmentación, por generaciones, permite comprender de una mejor manera a los grupos de turistas que comparten hábitos y comportamientos, que los diferencian entre otras generaciones y los hacen actuar, pensar y viajar de una manera distinta (Seabra et ál., 2020; Ahn et ál., 2019; Chung y Chen, 2019; Robinson y Schänzel, 2019; Beldona et ál., 2009).

Existen investigaciones realizadas en América, Europa y Asia que manifiestan que los gastos de los turistas con relación a sus viajes son diferentes entre generaciones (Ahn et ál., 2019; Wang y Davidson, 
2010; Li et ál., 2013); sin embargo, son escasas las investigaciones del gasto turístico de las cohortes generacionales que visitan el Ecuador y destinos específicos como Cuenca, ciudad declarada patrimonio cultural de la humanidad.

En este sentido, este trabajo tiene como objetivo analizar estas cohortes generacionales de turistas que visitan Cuenca, con un especial énfasis en el gasto turístico, para así aportar a la literatura empírica de forma que esta permita a las empresas públicas y privadas del sector turístico generar nuevas estrategias de mercado, comercialización en los segmentos de interés, mejorar la toma de decisiones y, sobre todo, generar productos innovadores que se adapten a cada segmento.

Para alcanzar nuestro objetivo el estudio se divide en secciones; la sección I, como se observa, corresponde a la introducción; la sección II detalla la revisión de los trabajos empíricos de las generaciones y el gasto turístico; la sección III detalla la metodología; la sección IV muestra los resultados, y la sección V presenta la discusión y conclusiones del estudio.

\section{2.}

\section{Revisión de la literatura empírica}

En primer lugar, es necesario comprender la frase: gasto turístico. Según el glosario de la Organización Mundial del Turismo (OMT) corresponde a "la suma pagada por la adquisición de bienes y servicios de consumo, y de objetos valiosos, para uso propio o para regalar, durante los viajes turísticos y para los mismos" (2008, p. 2).

Según las recomendaciones internacionales para estadísticas de turismo de la OMT, este gasto incluye:

Todos los bienes y servicios individuales considerados bienes o servicios de consumo en el Sistema de Cuentas Nacionales 2008 (aquellos que satisfacen los deseos y necesidades de las personas) pueden formar parte del gasto turístico. Éstos incluyen los servicios típicos adquiridos por los visitantes, como el transporte, el alojamiento, alimentos y bebidas, etcétera, pero también otros artículos, como objetos valiosos (pinturas, obras de arte, joyas, etcétera) con independencia de su valor unitario y adquiridos durante los viajes debido a su papel como depósitos de valor con el tiempo. (2008, p. 35)

También se toma en cuenta en el gasto, los bienes adquiridos para su utilización durante el viaje, como prendas de vestir específicas, medicamentos, etcétera; o artículos traídos como regalos para familiares y amigos. A esto se suma el hecho de que el gasto turístico no incluye valores correspondientes a pago de tasas e impuestos, intereses, adquisición de activos financieros y no financieros, adquisición de bienes con fines de reventa y todas las transferencias en efectivo, como donaciones a organizaciones benéficas o a otras personas (OMT, 2008).

Una de las primeras aproximaciones al término generación, desde el punto de vista sociológico, la realizó Mannheim (1993). En su libro manifiesta que:

La conexión generacional no es, ante todo, otra cosa que una modalidad especifica de posición de igualdad dentro del ámbito histórico social, debido a la proximidad de los años de nacimiento (...) la posición generacional se debe determinar a partir de ciertos momentos vitales que sugieren a los individuos afectados por ellos determinadas formas de vivencia y pensamiento. (p. 210)

Según Costanza et ál., (2012), el término generación se refiere a los "grupos de individuos basados en edades similares y experiencias compartidas. La idea es que las experiencias comunes compartidas por individuos de una edad en particular en un momento determinado crean similitudes entre los de la cohorte" (p.376).

Una aproximación más clara la da Díaz-Sarmiento et ál., (2017):

Cada generación tiene particularidades y rasgos característicos, diferencias en su fecha de nacimiento, se han enfrentado a contextos culturales, políticos y sociales diferentes y conciben las relaciones con sus empleadores y pares de una forma 
diferente. (...) Lo cual representa un gran reto para las organizaciones ya que esta diferencia impacta aspectos como comunicación, uso de la tecnología, motivación, reclutamiento, incentivos, entre otros. (p.193)

Los autores están de acuerdo con que no solo es un periodo de tiempo el que define la forma de actuar y de pensar de una generación, sino también sucesos e influencias del entorno. Además, sostienen que existen seis generaciones: Tradicionalistas, Generación Silenciosa, Baby Boomer, Generación X, Millennials y Centennials.

Otra noción importante es la de segmentación de mercado, como herramienta y componente fundamental de la mercadotecnia. Kotler y Keller (2012) explican que "la segmentación de mercado consiste en dividir el mercado en partes bien homogéneas, según sus gustos y necesidades. Un segmento de mercado consiste en un grupo de clientes que comparten un conjunto similar de necesidades y deseos" (p. 214).

Finalmente, es importante definir también el concepto de perfil turístico, pues es clave dentro de este estudio y se relaciona con lo expresado anteriormente. Según Pat y Calderón (2012) este se refiere a:

[...] la caracterización a un destino turístico. La caracterización con frecuencia se basa en variables socioeconómicas y demográficas de los turistas. El perfil permite conocer los gustos, preferencias, expectativas y necesidades de los turistas a efecto de mejorar la gestión de las empresas y los destinos turísticos. (p. 50)

\section{1 Características y preferencias de los turistas Baby Boomers}

La generación Baby Boomers surge entre los años de 1946 y 1964. Corresponde a quienes nacieron después del fin de la segunda guerra mundial. Esta época se caracterizó por el disparado incremento de niños, el Baby Boom, especialmente en países anglosajones (Díaz-Sarmiento et ál., 2017; Meredith et ál., 2003).

Los Baby Boomers están clasificados como inmigrantes digitales, pues nacieron en épocas anteriores al auge tecnológico de los últimos años. A pesar de que esta generación no creció con el uso del internet y de todas las herramientas tecnológicas con la que se cuenta en la actualidad, muchos de ellos se han adaptado a ellas de manera flexible en la vida laboral y personal (Li et ál., 2013).

De acuerdo con Beldona et ál., (2009), los Baby Boomers reportaron el mayor incremento agregado en el comportamiento de compras de viajes en línea" (p. 418). Esto sugiere que el uso de internet para adquirir servicios turísticos en este segmento ha aumentado mucho más que en otras generaciones, y que una parte importante de estos consumidores prefiere reservar sus viajes a través de Online Travel Agencies (OTAs). Según Tran (2018) los Baby Boomers:

Aprecian un sitio web de fácil uso que les permite reservar directamente con una empresa, por lo que es imperativo que los operadores turísticos y de actividades inviertan en un sitio web intuitivo que les facilite reservar uno de sus productos en línea. (p.1)

Según Li et ál., (2013), los Baby Boomers dan más importancia a los siguientes atributos: "alojamiento, calidad de servicio, amabilidad de las personas, facilidad para llegar al país, seguridad, valor para el dinero, información turística y limpieza" (p.160).

A esto se suma que el turista Baby Boomer se siente más motivado por actividades que cuentan con elementos culturales como: festividades locales, lugares históricos, experiencias de turismo comunitario, recorridos por el campo, entre otros; y prefieren, además, destinos como Sudamérica, África, América Central y Canadá (Li et ál., 2013). Es importante además mencionar que los Baby Boomers han generado incrementos en los comportamientos de compras de viajes en línea, en comparación con los turistas de la Generación X (Beldona et ál., 2009).

\subsection{Características y preferencias de los turistas de la Generación X}

Las personas de la Generación X son aquellas que nacieron entre los años 1978 y 1964 aproximadamente, son los hijos de los Baby Boomers, la mayor parte de ellos, padres de las nuevas generaciones (Kotler y Keller, 2012; Meredith et ál., 2003). 
Según Kotler y Keller (2012) "se considera que forman parte de una brecha intergeneracional; tienden puentes entre el conocimiento tecnológico de los $\mathrm{Mi}$ llenials y las realidades adultas de los Baby Boomers" (p. 220). Por lo que comparten algunas características con ambas generaciones.

Esta generación, en el momento de gastar, es más cautelosa y valora más la seguridad al adquirir productos o servicios. "La Generación X necesita pruebas convincentes de que un producto es confiable $\mathrm{y}$ simplificará su vida en lugar de complicarla" (Benckendorff et ál., 2009, p. 29). Por su parte, Ahn et ál., (2019) sostienen que la Generación X gasta más en recreación y comida que los Millennials.

Con respecto a la forma en la que viajan, Sevillano (2015) en su artículo Cuestión de generaciones, del periódico global "El País" señala:

Muchos tienen hijos menores e hipoteca (...) y tanto ella como él trabajan. Van a lo práctico, buscan buena relación calidad-precio y, más que unas caras vacaciones largas, hacen escapadas cortas. Improvisan más, planifican menos, y con menos antelación. Valoran la tecnología por su funcionalidad. Y piden viajes familiares y turismo de negocios.

\subsection{Características y preferencias de los turistas Millennials}

El término Millennials hace referencia a un rango etario o segmento de mercado que comprende a las personas nacidas entre los inicios de los años 80 y finales del siglo XX. Cualquier persona nacida desde 1980 hasta 1990 se encuentra en la Generación N, también denominada Millennial (Bernardi, 2018; Kotler y Keller, 2012 Gronbach, 2008). En tanto, la "Generación N" o "N-Gen" es la cohorte más joven; la llegada de Internet es un evento decisivo para ellos, y son el "motor" del crecimiento en las próximas dos décadas (Meredith et ál, 2003).

Según el reporte de la fuerza del turismo joven de la OMT (2011), en colaboración con la World Youth Student and Educational Travel Confederation, los "jóvenes representan aproximadamente 190 millones de viajes internacionales al año, y esta industria ha crecido con más rapidez que el turismo mundial. (...) los viajes anuales de jóvenes se cifrarán en casi 300 millones para 2020" (p. 6). La importancia de este crecimiento se ve reflejada en los ingresos que genera en los distintos países y ciudades destino.

Los viajeros jóvenes generaron unos ingresos, por turismo mundial, estimados en 165.000 millones de dólares, lo que confirma su valor financiero para la industria turística mundial y las economías locales. La fuerza del turismo joven no es sólo económica. Los beneficios sociales y culturales para el viajero joven y las comunidades que los reciben son de gran alcance, en el largo plazo, y considerablemente más sostenibles que otras formas de turismo (OMT, 2011).

Esta generación es muy variada en cuanto a sus hábitos y preferencias de consumo, sobre todo en relación a lo turístico. "El viajero Millennial no quiere llegar a destino y encontrarse con un show para turistas, sino que busca una atención personalizada e individualizada, y un viaje que esté adaptado a sus gustos y necesidades" (Allende, 2018, p. 55). Según el informe del viajero Millennial de Expedia, dentro de esta cohorte "hay diferencias significativas en por qué están eligiendo viajar; esto resulta en una amplia gama de aspiraciones de viaje, y por lo tanto, una amplia gama de tipos de vacaciones" (Future Foundation, 2016, p.3).

En el caso de los Millennials, la tecnología forma parte de su vida y no conciben una experiencia de viaje sin internet y sin su teléfono móvil. Los jóvenes hacen las reservas online, comparten sus opiniones en redes sociales y son los mayores usuarios de las plataformas de economía colaborativa (Allende, 2018, p. 47). En referencia al gasto, Ahn et ál., (2019) manifiestan que la generación Millennial tiende a gastar más en compras de recuerdos y productos locales que el Baby Boomer y la Generación X. Mientras su gasto en recuerdos y productos es mayor, Bernardi (2018) manifiesta que los turistas Millennials de Singapur e Indonesia, por ejemplo, buscan vuelos y alojamiento económicos.

Esta generación le da un mayor significado e importancia a los viajes y al turismo en comparación con sus generaciones predecesoras. Según el estudio de 
la OMT (2011) para los jóvenes "el turismo forma parte de su identidad - eres lo que has visitado" (p. 6). Los Millennials han impactado la forma de hacer turismo, pues viajan con el objetivo de tener una experiencia única. "En la actualidad, para la gente joven el turismo se ha convertido en algo que va más allá del hecho de viajar. El turismo consiste en vivir y disfrutar de experiencias de viaje genuinas y auténticas" (Allende, 2018, p. 47).

\section{3. \\ Metodología}

La presente investigación tiene un enfoque cuantitativo de alcance descriptivo, mediante un muestreo aleatorio simple, a tres segmentos de turistas: Baby Boomers, Generación X y los Millennials. El objetivo de la investigación es caracterizar el gasto turístico de manera comparativa con base en los perfiles turísticos por generación, con el fin de establecer diferencias y semejanzas entre estos.

Se estableció una muestra de 384 turistas con un margen de error de 5,5\% y un nivel de confianza del 95\%1 ${ }^{1}$. La muestra se estratificó de acuerdo con la clasificación de los grupos etarios presentada por el Ministerio de Turismo (2020). De acuerdo a estas cifras, existen 2.107.692 de llegadas de turistas al Ecuador en el año 2019. El 21\% corresponde a un rango etario de 20 a 29 años, de 30 a 39 años el 19,86\%, de 40 a 49 años el 16,43\%, de 50 a 59 años el 13,56\% y de 60 años y más con un porcentaje del $12,92 \%$; que en conjunto representan la mayor parte de los turistas que visitan el país con un 83,77\%. Estas edades corresponden a las generaciones: Baby Boomers, Generación X y Millennials, analizadas previamente en la revisión de la literatura. Se aplicaron 220 encuestas para turistas Millennials, 94 para turistas de la Generación X y 70 para turistas Baby Boomers.

Los encuestadores realizaron esta investigación en zonas de gran afluencia de turistas en la ciudad de Cuenca, como son el centro histórico, el aeropuerto Mariscal Lamar y el terminal terrestre, considerados sitios estratégicos para la realización de la investiga- ción, debido a que el primero es el núcleo del turismo en la ciudad; el segundo y el tercero son los principales puntos de arribo de los turistas.

Como principal instrumento para la recolección de datos se utilizó encuestas estructuradas, basadas en las recomendaciones internacionales para estadísticas de turismo de las Naciones Unidas y de la Organización Mundial de Turismo (OMT, 2008). Se realizó una encuesta para los tres segmentos de mercado, que contiene tres secciones diferentes. En la primera sección se recogió los datos de las características del visitante (edad, género, estado civil, situación laboral, ingresos); el segundo incluye preguntas sobre las características de los viajes turísticos (motivación principal, duración del viaje, medios de trasporte, entre otros); la última parte recoge la información referida al gasto turístico en la ciudad. Las encuestas son semejantes para los tres segmentos con excepción de las variables de motivación y el gasto diario por servicio. Los atributos de estas variables están basados en la revisión bibliográfica sobre hábitos de consumo y viaje de cada segmento de mercado.

Además de la encuesta, se realizaron ocho entrevistas no estructuradas a los grupos de turistas de cada generación, para obtener información y para agrupar las coincidencias de sus respuestas y construir las variables de la boleta.

Con la información recolectada, se realizó un análisis descriptivo de las variables de la encuesta en el programa de computación SPSS (Statistical Package for the Social Sciences), en el que se recolectaron tablas de frecuencias, porcentajes y medias, con el fin de profundizar en los objetivos planteados y contrastar relaciones entre las variables mediante tablas cruzadas. Finalmente, se analizó el gasto turístico a través del test estadístico ANOVA, que permite comparar la variable dependiente cuantitativa de gasto turístico, con la variable independiente categórica del tipo de generación. Además, se utilizó la prueba de comparaciones múltiples de Tukey para profundizar en la comparación de medias y determinar en dónde se encuentran las principales diferencias de gasto entre estos segmentos turísticos.

1 Según estimaciones de la Fundación de Turismo para Cuenca y de la Grupo de Investigación en Economía Regional de la Universidad de Cuenca en el año 2018 la cifra de turistas nacionales y extranjeros fue de 980.321 (Universidad de Cuenca, 2019). 


\section{4 . \\ Resultados}

Con base en la información recolectada, se realizó un análisis descriptivo de las características del visitante, de los viajes y del gasto turístico, por servicio (hospedaje, restauración, agencias de viaje, transporte y otros gastos) de los turistas que visitan la ciudad de Cuenca. A partir de esto, se generaron los perfiles de cada segmento, necesarios para el posterior análisis comparativo de su gasto.

\subsection{Perfil del turista Baby Boomer}

Los Baby Boomer, objeto de la muestra, son de ambos sexos (masculino 54\%, femenino 46\%), casados, retirados, jubilados; prefieren visitar la ciudad en pareja, poseen aproximadamente ingresos anuales de 50.000 dólares en promedio, son universitarios y con posgrado.

En cuanto a sus características de viaje turístico, se puede observar que sus principales motivaciones para visitar la ciudad de Cuenca son: la arquitectura, historia y el clima de la ciudad; otras motivaciones relevantes en esta generación son la comodidad de los servicios turísticos y la posibilidad de, en el futuro, residir en la ciudad de Cuenca.

Los turistas nacionales de esta generación proceden, principalmente, de ciudades del Ecuador como Guayaquil, Manta, Machala y Quito. Mientras que los extranjeros provienen principalmente de Estados Unidos y, en menor cantidad, de Francia y de Países Bajos. Con respecto al medio de transporte, este tipo de turista llega a la ciudad principalmente por vía aérea y planifica su viaje de manera directa.

Sobre las características del gasto turístico de esta generación en los servicios de hospedaje, alimentos, transporte, dentro de la ciudad y otros, se evidenció que generan un gasto menor a 30 dólares diarios en cada uno. Un dato importante es que esta generación no utiliza los servicios de una agencia de viajes. La mayor parte de este grupo tiene una estadía en la ciudad de tres días; sin embargo, una parte del segmento otorga importancia al hospedaje, cuando se hospedan en la ciudad por periodos de tiempo mayo- res a tres meses, debido a que consideran a la ciudad como un posible lugar de residencia.

Finalmente, el gasto diario total promedio de los turistas Baby Boomer que visitan la ciudad de Cuenca es de 85.75 dólares.

\subsection{Perfil del turista Generación X}

La Generación X, objeto de estudio, corresponde a turistas de ambos sexos (masculino 46,81\%, femenino 53,19\%), casados; prefieren visitar la ciudad en pareja y en familia; trabajan en el sector privado y son profesionales, con un nivel de ingreso anual menor a 20.000 dólares en promedio, son universitarios y con posgrado.

Conforme a las características de los viajes turísticos, visitan la ciudad de Cuenca motivados por su arquitectura e historia, la seguridad del destino y servicios turísticos de calidad. Se hospedan, principalmente, en hoteles y hacen uso de restaurantes y cafeterías como establecimientos de alimentación. La estadía de este segmento en la ciudad es, en promedio, de dos días, aunque un importante grupo de este segmento se queda hasta cinco días en la ciudad.

Los turistas de la Generación X son de nacionalidad extranjera, provienen de Estados Unidos y, en menor cantidad, de Perú e Inglaterra. Con respecto al turista nacional de este segmento, se evidencia que viene principalmente de ciudades como Guayaquil, Loja y Quito. Llegan a la ciudad por medio de transporte público o privado, y planifican su visita a la ciudad de Cuenca de manera directa y, en menor cantidad, a través del internet.

Con respecto al gasto diario de esta generación, los servicios de hospedaje y alimentación representan un rango de 21 a 40 dólares, en los servicios de transporte dentro de la ciudad gastan menos de 20 dólares y en otros gastos el gasto puede ascender a 40 dólares. Este segmento muestra un gasto no significativo en los servicios de agencias de viaje.

Por último, el gasto diario total de los turistas de la Generación X representa una media de 103,08 dólares. 


\subsection{Perfil del turista Millennial}

Con respecto a los turistas Millennials, se obtuvo que el 40\% son hombres y el 60\% mujeres. Algunos viajan en pareja, aunque, generalmente, lo hacen solos y en grupo o con amigos, en este orden; principalmente, son universitarios y con ingresos anuales menores a 20.000 dólares promedio.

Por otra parte, se encontró que dentro de las características de los viajes turísticos, visitan la ciudad motivados principalmente por el turismo de aventura, la interacción con los nativos y disfrutar de la gastronomía de la ciudad. El hospedaje de predilección de este segmento son los hostales, y hacen uso de restaurantes y plazas de comida como sus principales establecimientos de alimentación. Su estadía en la ciudad de Cuenca es de dos a cuatro días, pero la mayor parte del segmento utiliza tres días para visitar la ciudad y sus atractivos.

Proceden principalmente de países extranjeros como Alemania, Estados Unidos, Inglaterra y Países Bajos, pero el principal flujo de turistas proviene de Francia. El turista Millennial nacional es principalmente de las ciudades: Guayaquil, seguido de Quito, Loja y Babahoyo. Los turistas llegan a la ciudad a través del transporte público y planifican su visita a la ciudad de forma directa y a través del internet.

Por último, en cuanto al gasto turístico, los servicios de hospedaje, alimentación, transporte y otros gas- tos no sobrepasan los 10 dólares diarios. Dentro del servicio de agencias de viajes, no se registran gastos en este segmento. El promedio del gasto diario total que un Millennial genera en su visita a Cuenca es igual a 43,91 dólares.

\subsection{Análisis y comparación del gasto turístico}

Acorde con la caracterización de los perfiles de cada segmento, se ejecutó un análisis del gasto diario total de cada uno de ellos, seguido de las pruebas de análisis de la varianza -ANOVA- y Turkey, para el análisis de la relación entre este gasto y la generación a la que el turista pertenece.

La Figura 1 señala que el promedio de gasto de los turistas Baby Boomers es igual a 85,70 dólares al día, el valor mínimo que un turista gasta es de 10,23 dólares mientras que el valor máximo es de 500 dólares. $\mathrm{El}$ valor con mayor frecuencia correspondiente a la moda es igual a 50 dólares y de 100 dólares. Finalmente, la desviación estándar es de 80,11 dólares.

La Figura 2 evidencia que el gasto diario promedio del turista de la Generación X es igual a 103,10 dólares al día, el valor mínimo que un turista gasta es de 3,33 dólares, mientras que el valor máximo es de 500 dólares La moda estadística de este segmento de mercado es 100 dólares y la deviación estándar es de 83,02 dólares.

\section{Figura 1}

Gasto diario total del turista Baby Boomer que visita Cuenca (en dólares)

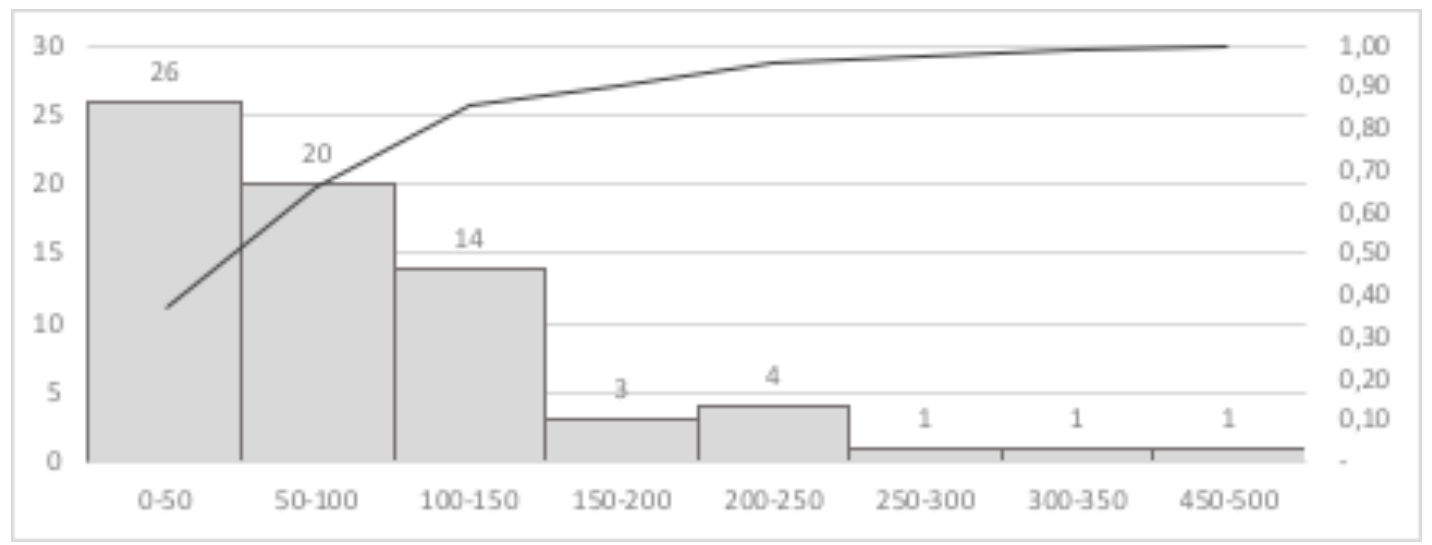

Nota: Media $=85.70$, DesvStd. $=80.11, \mathrm{~N}=70$. 


\section{Figura 2}

Gasto diario total del turista Generación X que visita Cuenca (en dólares)

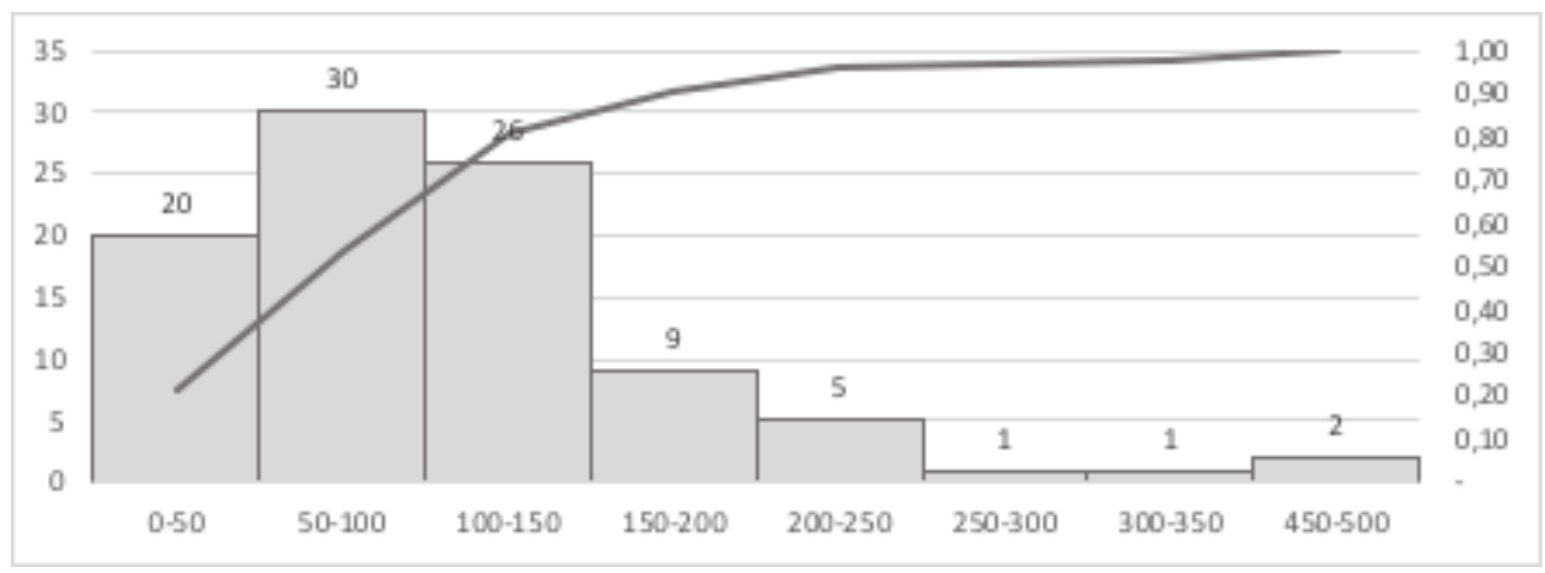

Nota: Media $=103.10$, DesvStd. $=83.02, \mathrm{~N}=94$.

\section{Figura 3}

Gasto diario total del turista Millennial que visita la ciudad de Cuenca (en dólares)

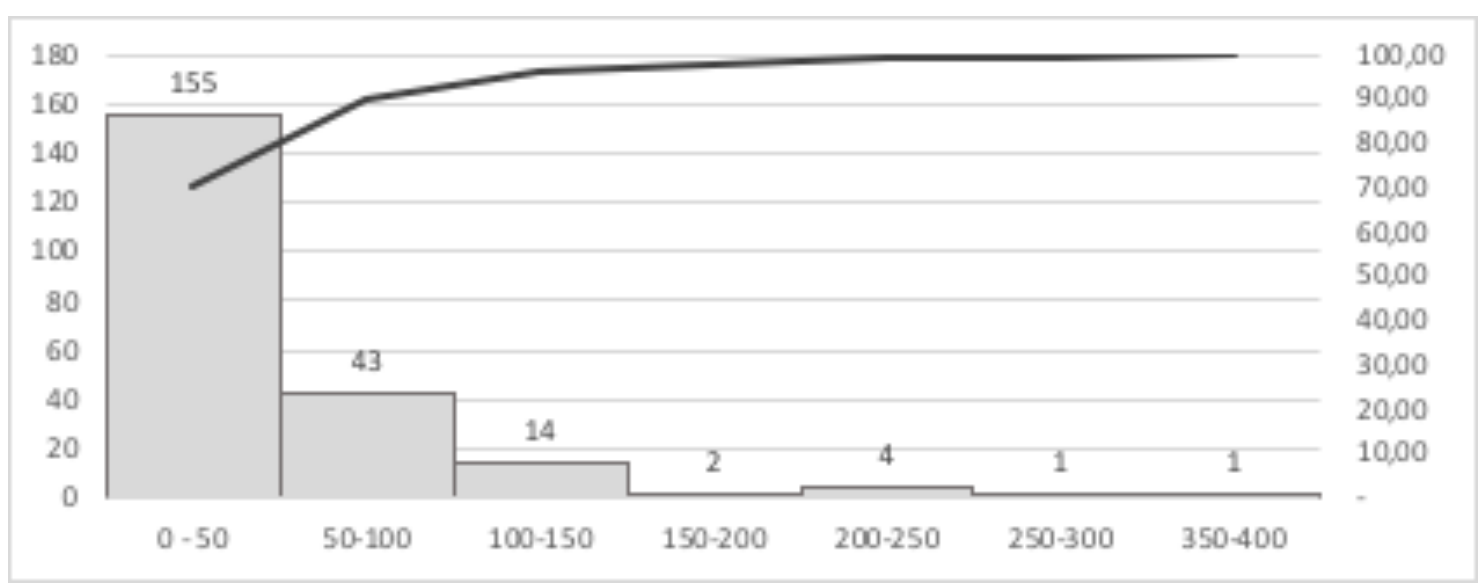

Nota: Media $=43.90$, DesvStd. $=45.43, \mathrm{~N}=220$

La Figura 3 muestra que el promedio de gasto de un turista Millennial es igual a 43,91 dólares al día, el valor mínimo que un turista gasta es de 2,33 dólares, mientras que el valor máximo es de 350 dólares. El valor con mayor frecuencia correspondiente a la moda es igual a 50 dólares. La deviación estándar es de 45,43 dólares.

\subsection{Análisis de varianza ANOVA}

Finalmente, se realizó el test estadístico ANOVA de un factor; el cual involucra una variable independiente categórica y una variable dependiente cuantitativa, con el fin de contrastar la hipótesis de igualdad entre las medias o promedios de la variable cuantitativa, con relación a caga grupo de la variable independiente o factor, y demostrar si existe una diferencia estadística significativa entre estos.

En este sentido, se busca comparar la relación entre la variable generación -Baby Boomers, Generación X o Millennials- como factor, con el gasto diario total de los turistas, con el fin de encontrar diferencias en el gasto turístico entre los turistas. 


\section{Tabla 1}

Análisis descriptivo del gasto turístico diario total (en dólares) por generación

\begin{tabular}{|l|c|c|c|c|c|c|c|c|}
\hline & N & Media & $\begin{array}{c}\text { Desviación } \\
\text { Estándar }\end{array}$ & $\begin{array}{c}\text { Error } \\
\text { Estándar }\end{array}$ & $\begin{array}{c}\text { Límite } \\
\text { Inferior }\end{array}$ & $\begin{array}{c}\text { Límite } \\
\text { Superior }\end{array}$ & Mínimo & Máximo \\
\hline Baby Boomers & 70 & 85.75 & 80.11 & 9.57 & 66.55 & 104.85 & 10.2 & 500.0 \\
\hline Generación X & 94 & 103.08 & 83.02 & 8.56 & 86.07 & 120.08 & 3.3 & 500.0 \\
\hline Millennials & 220 & 43.91 & 45.43 & 3.06 & 37.87 & 49.94 & 2.3 & 350.0 \\
\hline Total & 384 & 66.02 & 68.55 & 3.50 & 59.14 & 72.90 & 2.3 & 500.0 \\
\hline
\end{tabular}

La Tabla 1 evidencia un análisis descriptivo de la variable gasto turístico dentro de cada generación y se observa que los Baby Boomers tienen una media de 85.75 dólares, la Generación X de 103.08 dólares y los Millennials de 43.91 dólares. También se observa en la Tabla 1 la frecuencia, desviación y error estándar, así como los intervalos de confianza y los valores mínimos y máximos dentro de cada segmento de mercado.

\section{Tabla 2}

Análisis de varianza ANOVA

\begin{tabular}{|l|c|c|c|c|c|}
\hline & $\begin{array}{c}\text { Suma de } \\
\text { Cuadrados }\end{array}$ & Df & $\begin{array}{c}\text { Cuadrado } \\
\text { medio }\end{array}$ & F & Significancia \\
\hline Entre grupos & 263929.66 & 2 & 131964.83 & 32.74 & $0,000^{*}$ \\
\hline Intragrupos & 1535711.51 & 381 & 4030,74 & & \\
\hline Total & 1799641.17 & 383 & & & \\
\hline
\end{tabular}

Nota: *significancia al 0,05

La Tabla 2 muestra los resultados del análisis de varianza ANOVA, donde el estadístico F igual a 32.74 y el valor de significancia igual a 0.000 inferior al nivel de significación del $5 \%$, de esta manera se rechaza la hipótesis nula, y se afirma que existe una diferencia estadística significativa entre las medias de estos segmentos de mercado.

Después de realizar el test estadístico ANOVA y rechazar la hipótesis nula, se empleó la prueba post hoc Tukey que permite realizar comparaciones múltiples entre los segmentos Baby Boomers, Generación X y Millennials, con el objetivo de encontrar diferencias estadísticas de una manera profunda. 


\section{Tabla 3}

Comparaciones múltiples-Prueba Estadística Tukey

\begin{tabular}{|c|c|c|c|c|c|c|}
\hline \multicolumn{7}{|c|}{ Comparaciones múltiples } \\
\hline \multicolumn{7}{|c|}{ Variable dependiente: Variable dependiente: Gasto Diario Total } \\
\hline \multirow{2}{*}{$\begin{array}{c}\text { (I) } \\
\text { Generación }\end{array}$} & \multirow{2}{*}{$\begin{array}{c}\text { (J) } \\
\text { Generación }\end{array}$} & \multirow{2}{*}{$\begin{array}{l}\text { Diferencia } \\
\text { de medias } \\
\quad(\mathrm{I}-\mathrm{J})\end{array}$} & \multirow{2}{*}{$\begin{array}{c}\text { Error } \\
\text { estándar }\end{array}$} & \multirow{2}{*}{ Sig. } & \multicolumn{2}{|c|}{$\begin{array}{l}95 \% \text { de intervalo de } \\
\text { confianza }\end{array}$} \\
\hline & & & & & $\begin{array}{l}\text { Límite } \\
\text { inferior }\end{array}$ & $\begin{array}{l}\text { Límite } \\
\text { superior }\end{array}$ \\
\hline \multirow{2}{*}{$\begin{array}{l}\text { Baby } \\
\text { Boomers }\end{array}$} & Generación X & $-17,3322$ & 10,0231 & ,196 & $-40,916$ & 6,251 \\
\hline & Millennial & $41,8415^{*}$ & 8,7123 & ,000 & 21,342 & 62,341 \\
\hline \multirow{2}{*}{$\begin{array}{l}\text { Generación } \\
X\end{array}$} & Baby Boomer & 17,3322 & 10,0231 & ,196 & $-6,251$ & 40,916 \\
\hline & Millennial & $59,1736 *$ & 7,8232 & ,000 & 40,766 & 77,581 \\
\hline \multirow{2}{*}{ Millennials } & Baby Boomer & $-41,8415^{*}$ & 8,7123 &, 000 & $-62,341$ & $-21,342$ \\
\hline & Generación X & $-59,1736^{*}$ & 7,8232 & ,000 & $-77,581$ & $-40,766$ \\
\hline
\end{tabular}

Nota: *significancia al 0,05

En la Tabla 3 se observan las comparaciones múltiples entre las generaciones: el segmento Baby Boomers no tiene una diferencia estadísticamente significativa con la Generación X, por lo que se acepta la hipótesis nula de igualdad de promedios de gasto turístico, mientras que con el segmento Millennials existe una diferencia estadística. De igual manera, la Generación X y Millennials presentan una diferencia estadística entre sus promedios de gasto turístico.
La Tabla 4 presenta la media del gasto diario total de todas las generaciones, los Millennials difieren de forma estadísticamente significativa con la media del gasto diario total de los Baby Boomers y Generación $\mathrm{X}$, lo que revela dos subconjuntos diferentes.

\section{Tabla 4}

Subconjuntos Homogéneos-Prueba Estadística Tukey

\begin{tabular}{|l|c|c|c|}
\hline \multicolumn{4}{|c|}{ Gasto Diario Total } \\
\hline HSD Tukey & \multicolumn{3}{|c|}{ Subconjunto para alfa = $\mathbf{0 . 0 5}$} \\
\hline Generación & $\mathrm{N}$ & 1 & 2 \\
\hline Millennials & 220 & 43,905 & \\
\hline Baby Boomers & 70 & & 85,747 \\
\hline Generación X & 24 & & 103,079 \\
\hline Sig. & & 1,000 &, 127 \\
\hline
\end{tabular}


5.

\section{Discusión y Conclusiones}

El objetivo de este estudio fue comparar el gasto turístico en tres segmentos de mercado -Baby Boomer, Generación X y Millennial- que visitan la ciudad de Cuenca-Ecuador.

Se evidenció que la generación de los Baby Boomer otorga importancia al hospedaje, debido a que se alojan en la ciudad por periodos de tiempo largos. Un enunciado semejante al de Li, et ál., (2013) que consideran que los Baby Boomers otorgan mayor importancia al alojamiento. La Generación X asigna un presupuesto de viaje menor para el transporte y mayor para hospedaje y alimentación, hallazgos que coinciden con Ahn et ál., (2019). La generación Millennial asigna una parte mínima de su presupuesto de viaje para los rubros restauración, transporte y alojamiento, sin utilizar el servicio de agencias de viajes, hecho que coincide con los resultados de Bernardi (2018) que manifiesta que los Millennials buscan soluciones más económicas.

Además, las actividades de atracción turística de estas generaciones muestran diferentes niveles de participación en el destino. La motivación turística de los Baby Boomers y la Generación X es la arquitectura y la historia de la ciudad; a los Millennials, por otro lado, les interesa el turismo de aventura, la interacción con los nativos y disfrutar de la gastronomía de la ciudad. La Generación X es el segmento con una mayor media de gasto diario total, con una menor duración de viaje, mientras que la estadía de los Millennials y del Baby Boomer es mayor.

Por otra parte, se comprobó que existe una diferencia estadística significativa entre los promedios del gasto turístico y el segmento generacional de turistas, hallazgo que se corrobora con lo planteado por Ahn et ál., (2019), que confirman una diferencia en los patrones de gasto y los segmentos de cohorte generacional.

Luego de lo expuesto, los hallazgos de este estudio permiten contribuir a la literatura empírica sobre los perfiles de los turistas de cada segmento y su gasto durante su visita a ciudades de países latinoamericanos como el Ecuador y en un destino específico como Cuenca.
La investigación aporta, además, al conocimiento del gasto de viaje que realizan los turistas en un destino, lo que permite generar estrategias de precio para los productos y los servicios turísticos, con base en su generación, además de políticas de desarrollo para maximizar los beneficios que provienen del turismo. A esto se suma, la generación de estrategias de precio para los productos y servicios turísticos con base en los presupuestos de viaje de cada generación. Las limitaciones del estudio se encontraron en la revisión de la literatura empírica sobre el gasto turístico entre generaciones y la delimitación de las edades de las generaciones, sin embargo, esta investigación aporta datos representativos que demuestran una diferencia en los hábitos de gasto entre los turistas de distintas generaciones, lo que sugiere estrategias diferenciadas por parte de la empresas del sector turístico sobre el patrón del presupuesto que considere cohortes generacionales.

Las líneas de investigación futuras se relacionan con el estudio de las personalidades de cada una de las generaciones, sus características y su influencia en el turismo dentro de la ciudad de Cuenca, así como futuros análisis del gasto turístico por temporada y enfocado, específicamente, a cada uno de estos segmentos.

\section{6.}

\section{Referencias}

Ahn, Y. J., Lee, B. C., y Lee, S. K. (2019). Analysis of Korean millennials' travel expenditure patterns: an almost ideal demand system approach. Asia Pacific Journal of Tourism Research, 25(1), 3-14. https://doi.org/10.1080/10941665.2019.1578810

Allende, E. (2018). Jóvenes y hábitos de consumo en turismo. Revista de Estudios de Juventud. 119 (5), 1-15. Disponible en: https://n9.cl/16qs

Beldona, S., Nusair, K., y Demicco, F. (2009). Online travel purchase behavior of generational cohorts: a longitudinal study. Journal of Hospitality Marketing \& Management, 18(4), 406-420. https://doi.org/10.1080/19368620902799627 
Benckendorff, P., Moscardo, G. y Pendergast, D. (2009). Tourism and Generation Y. London: CIB International.

Bernardi, M. (2018). Millennials, sharing economy and tourism: the case of Seoul. Journal of Tourism Futures, 4(1), 43-56.

https://doi.org/10.1108/jtf-12-2017-0055

Brida, J. G., Y Scuderi, R. (2013). Determinants of tourist expenditure: A review of microeconometric models. Tourism Management Perspectives, 6, 28-40. https://doi.org/10.2139/ssrn.2048221

Costanza, D. P., Badger, J. M., Fraser, R. L., Severt, J. B., y Gade, P. A. (2012). Generational Differences in Work-Related Attitudes: A Meta-analysis. Journal of Business and Psychology, 27(4), 375-394. https://doi.org/10.1007/s10869-012-9259-4

Chung, J. Y., \& Chen, C. C. (2019). Generational differences in international stereotypes and destination images: tourism between partitioned states. Journal of Travel \& Tourism Marketing, 36(8), 865-876.

https://doi.org/10.1080/10548408.2019.1657051

Díaz-Sarmiento, C., López-Lambraño M. y Roncallo-Lafont, L. (2017). Entendiendo las generaciones: una revisión del concepto, clasificación y características distintivas de los Baby Boomers, X y Millennials. Clío América, 11(22), 188204. https://doi.10.21676/23897848.2440

Future Foundation (2016). Millennial traveller report why Millennials will shape the next 20 years of travel, Expedia. Consultado el 15 de septiembre de 2019 en https://n9.cl/2mdp

Gronbach, K. W. (2008). The age curve: How to profit from the coming demographic storm. New York: Amacom, p. 288.

Kotler, P. \& Keller, K. (2012). Dirección de Marketing. México: Pearson Education Inc.
Li, X., Li, X. R., y Hudson, S. (2013). The application of generational theory to tourism consumer behavior: An American perspective. Tourism Management, 37, 147-164.

Mannheim, K. (1993). El problema de las generaciones. Reis, (62), 193-244. Disponible en https://bit.ly/2XXOoEO

Meredith, G. E., Schewe, C. D., \& Karlovich, J. (2003). Defining markets, defining moments: America's 7 generational cohorts, their shared experiences, and why businesses should care, Journal of Consumer Marketing, 20(2), 172-173. https://doi.org/10.1108/07363760310464622

Ministerio de Turismo (2020). Turismo en Cifras. Consultado el 11 de mayo de 2020 en https://n9.cl/tn3j

OMT-Organización Mundial del Turismo y Naciones Unidas (2008). Recomendaciones Internacionales para estadísticas de turismo 2008. Consultado el día 3 de diciembre en http://www2.unwto.org/es

OMT-Organización Mundial del Turismo (2011). La fuerza del turismo joven. Consultado el 4 de septiembre de 2019 en https://n9.cl/j5lq

Pat, L., y Calderón, G. (2012). Caracterización del perfil turístico en un destino emergente, caso de estudio de ciudad del Carmen, Campeche. México, Gestión Turística, 2, 47-70. https://bit.ly/2zyBgLa

Robinson, V. M., y Schänzel, H. A. (2019). A Tourism Inflex: Generation $\mathrm{Z}$ travel experiences. Journal of Tourism Futures, 5 (2), 127-141. https://doi.org/10.1108/JTF-01-2019-0014

Seabra, C., Pereira, A., Silva, C., Abrantes, J. L., Reis, M., \& Paiva, O. (2020). Destination image perceived by domestic tourists: The influence of Generation Gap. European Journal of Tourism Research, 25, 1-22. 
Sevillano, E. (2015). El País periódico global. Consultadoel 8 de agosto de 2019 en:

https://bit.ly/2ABF5Qf

Tran, L. (2018). Baby Boomers: An In-depth Look into the Travel Segment, Rezdy. Consultado el 19 de diciembre de 2019 en

https://bit.ly/3dVvW3n

Universidad de Cuenca (2019). Estudio de la demanda y Oferta Turística de la ciudad de Cuenca Boletín Anual, año 2018.Consultado el 11 de enero de 2020 en https://bit.ly/2UETim4

Wang, Y., \& Davidson, M. C. (2010). A review of micro-analyses of tourist expenditure. Current Issues in Tourism, 13(6), 507-524.

https://bit.ly/2Ap6FQL 
